\title{
Physiologically Realistic Formation of Autoassociative Memory in Networks with Theta/Gamma Oscillations: Role of Fast NMDA Channels
}

\author{
Ole Jensen, ${ }^{1}$ Marco A.P. Idiart, ${ }^{2}$ and John E. Lisman ${ }^{1,3}$ \\ ${ }^{1}$ Volen Center for Complex Systems \\ Brandeis University \\ Waltham, Massachusetts 02254 \\ ${ }^{2}$ Instituto Fisica \\ UFRGS \\ 91501-970 Porto Alegre, RS Brazil
}

\begin{abstract}
Recordings from brain regions involved in memory function show dual oscillations in which each cycle of a low-frequency theta oscillation $(5-8 \mathrm{~Hz})$ is subdivided into about seven subcycles by high frequency gamma oscillations $(20-60 \mathrm{~Hz})$. It has been proposed (Lisman and Idiart 1995) that such networks are a multiplexed short-term memory (STM) buffer that can actively maintain about seven memories, a capability of human STM. A memory is encoded by a subset of principal neurons that fire synchronously in a particular gamma subcycle. Firing is maintained by a membrane process intrinsic to each cell. We now extend this model by incorporating recurrent connections with modifiable synapses to store long-term memory (LTM). The repetition provided by STM gradually modifies synapses in a physiologically realistic way. Because different memories are active in different gamma subcycles, the formation of autoassociative LTM requires that synaptic modification depend on $\boldsymbol{N}$-methyl-D-aspartate (NMDA) channels having a time constant of deactivation that is of the same order as the duration of a gamma subcycle (15-50 msec). Many types of NMDA channels have longer time constants (150 msec), as for instance those found in the hippocampus, but both fast and slow NMDA channels are present in

cortex. This is the first proposal for the special role of these fast NMDA channels. The STM for novel items must depend on activity-dependent changes intrinsic to neurons rather than recurrent connections, which have not developed the required selectivity. Because these intrinsic mechanisms are not error-correcting, STM will become slowly corrupted by noise. This limits the accuracy with which LTM can become encoded after a single presentation. Accurate encoding of items in LTM can be achieved by multiple presentations, provided different memory items are presented in a varied interleaved order. Our results indicate that a limited memory-capacity STM model can be integrated in the same network with a high-capacity LTM model.

\section{Introduction}

Insights into the mechanisms of memory have come from several different fields. Psychophysical work indicates the existence of a short-term memory (STM) mechanism of limited capacity ( $7 \pm 2$ items) (Miller 1956) and a long-term memory (LTM) mechanism of much higher capacity. Physiological work indicates that STM is maintained by persistent cell firing (Fuster and Jervey 1982; Goldmann-Rakic 1995), whereas LTM appears to be maintained by modifications of synaptic strength (Bliss and Collingridge 1993; Morris et al. 1986). Theoretical analysis of neural networks has indicated how recurrent collaterals with modifiable synapses can lead to distributed storage of

LEARNING \& MEMORY 3:243-256 @ 1996 by Cold Spring Harbor Laboratory Press ISSN1072-0502/96 $\$ 5.00$

$$
\begin{array}{lllllllll}
L & E & A & R & N & I & N & G & \mathbf{Q} \\
\mathbf{2 4 3} & M & E & M & O & R & Y
\end{array}
$$


LTM (Hopfield 1982; Amit 1989; Hertz et al. 1991). Most recently it has been found that networks involved in memory storage show complex oscillatory dynamics. The hippocampus and some regions of cortex have dual oscillations in which a low-frequency oscillation in the theta frequency range $(5-8 \mathrm{~Hz})$ is subdivided into subcycles by a high-frequency oscillation in the gamma (20-60 $\mathrm{Hz}$ ) range (Biedenbach 1966; Bressler 1984; Soltesz and Deschenes 1993; Bragin et al. 1995).

We have been interested in synthesizing the results from these fields. Our initial work focused on the relationship between network oscillations and the psychophysical properties of STM (Lisman and Idiart 1995). We proposed that dual oscillations serve as a multiplexing mechanism by which multiple short-term memories can be actively maintained in the same network. A STM is assumed to be encoded by a subset of cells that fire synchronously during a given gamma subcycle, an assumption consistent with previous work indicating the importance of synchrony (Gray et al. 1989; Abeles et al. 1993). Because there are about seven gamma cycles in a theta cycle, this could explain why STM is limited to seven items. Further support for this view is that the Sternberg number (Sternberg 1966), which is a psychophysical measurement of the temporal separation of memories, approximately equals the period of a gamma oscillation. The proposition that different information is stored at different phases of a theta cycle (i.e., in different gamma subcycles) is made plausible by studies of hippocampal place cells (O'Keefe and Recce 1993; Skaggs et al. 1996) showing that different cells fire with different phases during the theta cycle.

A second feature of our previous model is the mechanism by which the firing that underlies STM is maintained. Action potentials triggered at the time of information input to the network trigger an after-depolarization (ADP) that is intrinsic to each neuron (Lisman and Idiart 1995). An ADP of this kind has been observed in hippocampal and cortical neurons (Andrade 1991; Caeser et al. 1993; Storm 1989; Libri et al. 1994). The ADP has sufficient duration to trigger firing on subsequent theta cycles and thereby maintains STM. This mechanism for maintaining firing stands in contrast to reverbatory mechanisms involving loops of interconnected cells (Hebb 1949). In our previous STM model (Lisman and Idiart 1995) there was no mechanism for LTM (Fig. 1A). In contrast standard neural network models (Hopfield 1982; Amit
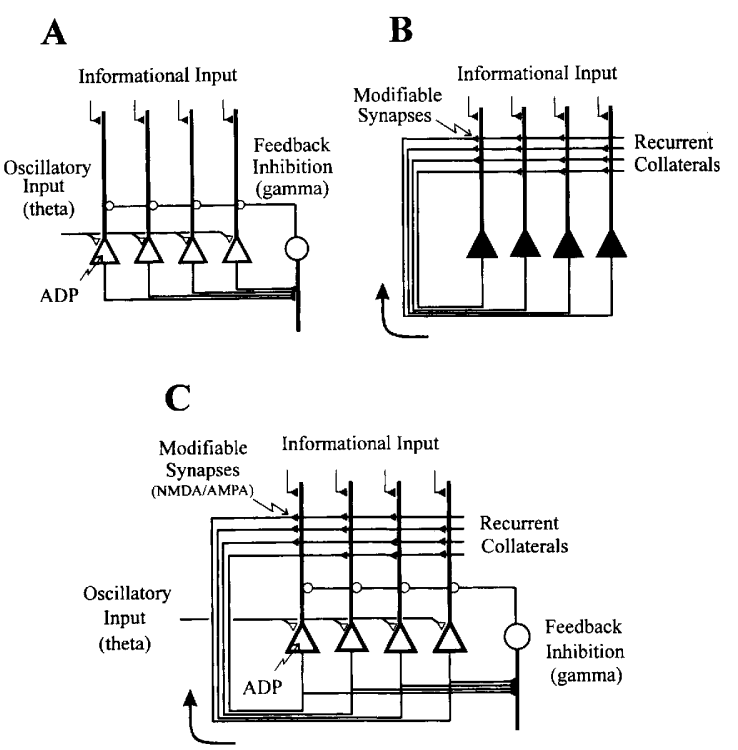

Figure 1: The goal of this series of papers is to examine networks that integrate the STM and LTM networks. (A) STM network in which information is stored using the ADP that is intrinsic to each pyramidal cell. Dual oscillations occur because of external theta-frequency input and because of feedback inhibition from an inhibitory interneuron, which generates the gamma cycles. (B) Autoassociative network in which LTM is encoded in modifiable recurrent synapses. (C) Integrated network that has both STM and LTM. Synaptic modification is governed by fast NMDA channels. Synaptic transmission is mediated by AMPA channels.

1989; Hertz et al. 1991 ) have recurrent collaterals with modifiable synapses (Fig. 1B) for storing LTM, but no mechanism for storing novel STMs. Our goal in this and the subsequent papers in this series is to examine networks that have both STM and LTM. As shown in Figure 1C, the ADP feature of our STM model and the modifiable synapses of standard LTM models are compatible and integrated models can be analyzed.

By studying the interaction of STM and LTM, we have attempted to capture temporal complexities of real learning situations and the kinetic properties of the cellular processes that underlie learning. It is a general assumption of our work that synaptic modification similar to long-term potentiation (LTP) and long-term depression (LTD) underlies the storage of LTM (Morris et al. 1986), even though this is not firmly established. Experimental evidence indicates that LTP occurs slowly and requires repetitive firing (Larson et al. 1986; Hanse and Gustafsson 1994). Specifically, experiments on hippocampal LTP show that induction

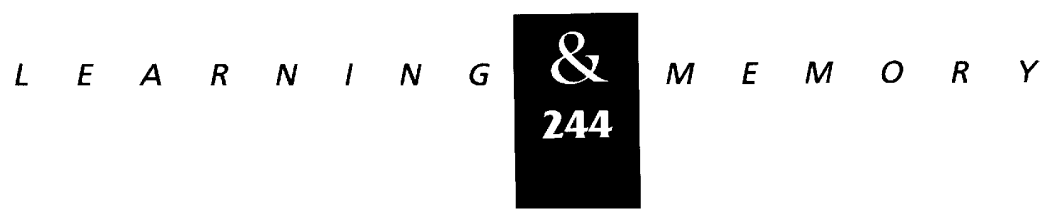


requires a second or more high-frequency stimulation under standard conditions (Bliss and Lømo 1973). Once LTP has been induced it takes additional time (tens of seconds) to express itself (Hanse and Gustafsson 1994). Thus a STM buffer is needed both to cause the persistent firing required for transfer of information to LTM and to maintain information until LTM is expressed. An additional complexity is that memory items often arrive in streams of multiple items per second rather than as single items. An STM buffer that can capture multiple items and actively maintain them is capable of dealing with such streams.

In this paper we deal specifically with the learning of novel items. An example would be the first time one is exposed to a digit. We show that this novelty creates special problems for STM and the correct formation of autoassociative LTM. In a different class of STM tasks, the items themselves are already known (e.g., the digits); what has to be remembered is which items are present and in what order, as for instance in a phone number. The fact that the individual items are familiar implies that they are already stored autoassociatively in LTM. In the second paper in this series (Jensen and Lisman 1996b, this issue) we show how these representations in LTM can dramatically enhance the reliability with which arbitrary lists of such items can be stored in STM.

A critical insight to emerge from our analysis of networks with theta/gamma oscillations regards the importance of the kinetics of $N$-methyl-D-aspartate (NMDA) channels. These channels control the induction of LTP at Hebbian synapses (for review, see Bliss and Collingridge 1993). Recent work shows that different subtypes of the NMDA channel have very different time constants of deactivation (Hestrin et al. 1994; Monyer 1994; S. Vicini, J.F. Wang, J.S. Poling, and D.R. Grayson, pers. comm. ). Fast NMDA channels have a time constant on the same scale as a gamma cycle, but other isoforms have much longer time constants. In this paper we show that fast NMDA channels $(\tau \sim 25$ msec) have kinetics that are appropriate for the formation of autoassociative LTM. We show that NMDA channels with a slow time constant for deactivation $(\tau \sim 150 \mathrm{msec})$ would not be well suited for forming autoassociative memories, but are ideally suited for forming heteroassociative memory sequences. In the third paper in this series (Jensen and Lisman 1996c, this issue), we show how networks with slow NMDA channels can form LTM for sequences of known items (e.g., a phone number). We show that learning sequences of known items can occur after a single presentation. Therefore, this is a model of episodic memory. Because hippocampal synapses have only slow NMDA channels (Forsythe and Westbrook 1988; Jahr and Stevens 1990), an implication is that the hippocampal $\mathrm{CA} 3$ region, long thought to be an autoassociative network, may actually be a heteroassociative network specialized for sequence learning. The ability of such heteroassociative models to quantitatively account for important properties of hippocampal place cells is described in a final paper in this series (Jensen and Lisman 1996a, this issue).

\section{Materials and Methods}

The field of neuronal modeling spans from implementation of abstract binary neurons to detailed compartmental models. An intermediate approach has been used here. The neurons are simplified to have a single compartment, but important temporal characteristics of some membrane processes are explicitly modeled. We have implemented the network using spiking model neurons so that STM can be encoded by synchrony of spikes rather than by rates (Gerstner et al. 1993; Hopfield and Hertz 1995).

The architecture of the network is illustrated in Figure 1C. The net current to each pyramidal neuron in the network is approximated by

$$
i_{\text {net }}=i_{\text {leak }}+i_{A D P}+\mathrm{i}_{A}+i_{\text {tbeta }}+i_{G A B A}+i_{\text {syn }}+i_{\text {ext }}
$$

For simplicity we have ignored the properties of the membrane capacitance. The main effect of the capacitance is to slow down transients and to filter noise. Because we have not modeled the membrane capacitance explicitly we have slightly slowed the kinetics of synaptic currents, so that the resulting synaptic voltage events have reasonable kinetics.

$$
\begin{aligned}
V(t)= & R_{\text {membrane }}\left(i_{A D P}(t)+i_{A}(t)+i_{\text {theta }}(t)+i_{G A B A}(t)\right. \\
& \left.+i_{\text {syn }}(t)+i_{\text {ext }}(t)\right)+V_{\text {rest }}
\end{aligned}
$$

The average input resistance in pyramidal CA3 cells has been found to be $\sim \mathbf{R}_{\text {membrane }}=33 \mathrm{M} \Omega$ (Turner and Schwartzkroin 1983).

SPIKING

We have not implemented the fast currents involved in action potentials and bursting. A spike

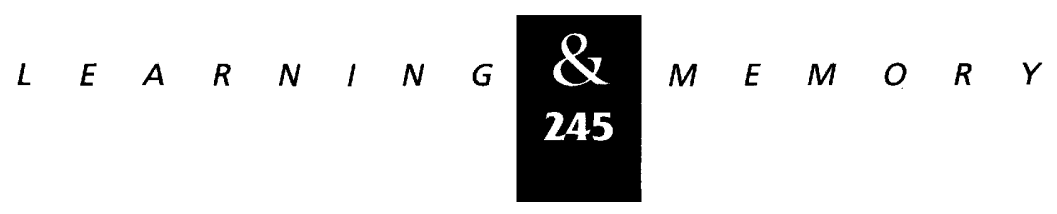


Jensen et al.

is modeled as an instantaneous event that occurs when threshold is reached, $V_{\text {thres }}=-50 \mathrm{mV}$. The cell is then reset to $V_{\text {rest }}=-60 \mathrm{mV}$.

\section{AFTER-DEPOLARIZATION (ADP)}

Pyramidal cells exhibit an after-depolarization (ADP) after spiking during cholinergic (Storm 1989; Andrade 1991; Caeser et al. 1993; Libri et al. 1994) or serotonergic modulation (Araneda and Andrade 1991). The ADP provides a ramp of excitation that builds up after a spike, within a time of $200 \mathrm{msec}$ and then falls. We model $i_{A D P}$ by an alpha function:

$$
i_{A D P}(t)=A_{A D P} \frac{t-t_{\text {fire }}}{\tau_{A D P}} \exp \left(1-\frac{t-t_{\text {fire }}}{\tau_{A D P}}\right)
$$

Whenever the cell spikes again, the ADP is reset and starts to build up again. We have used $\tau_{A D P}=200 \mathrm{msec}$. This means that the ADP starts to decline just after a subsequent theta cycle unless the ADP is refreshed. It is this decline that underlies the dropout of a previously inserted memory when an eighth memory is introduced (see Fig. 5 of Jensen and Lisman 1996b, this issue). A consequence of picking the $\tau$ of the ADP in this way is that the ADP is fairly flat (Fig. 2c) at the time when the ADP triggers spiking. It is for this reason that the timing ramps provided by the ADP can be disrupted by low levels of noise. We suspect that a more complex and realistic model of the ADP would allow more realistic choices of membrane noise.

\section{FAST AFTER-HYPERPOLARIZATION (AHP)}

The fast AHP current prevents pyramidal cells from fast, repetitive firing. We model the current by a decreasing exponential:

$$
i_{A}(t)=A_{A} \exp \left(-\frac{t-t_{\text {fire }}}{\tau_{A}}\right)
$$

where $\tau_{A}=5 \mathrm{msec}$ (see Storm 1990 for approximate values).

\section{THETA OSCILLATIONS}

Theta oscillations have been identified in the hippocampus (Vanderwolf 1969; Stewart and Fox
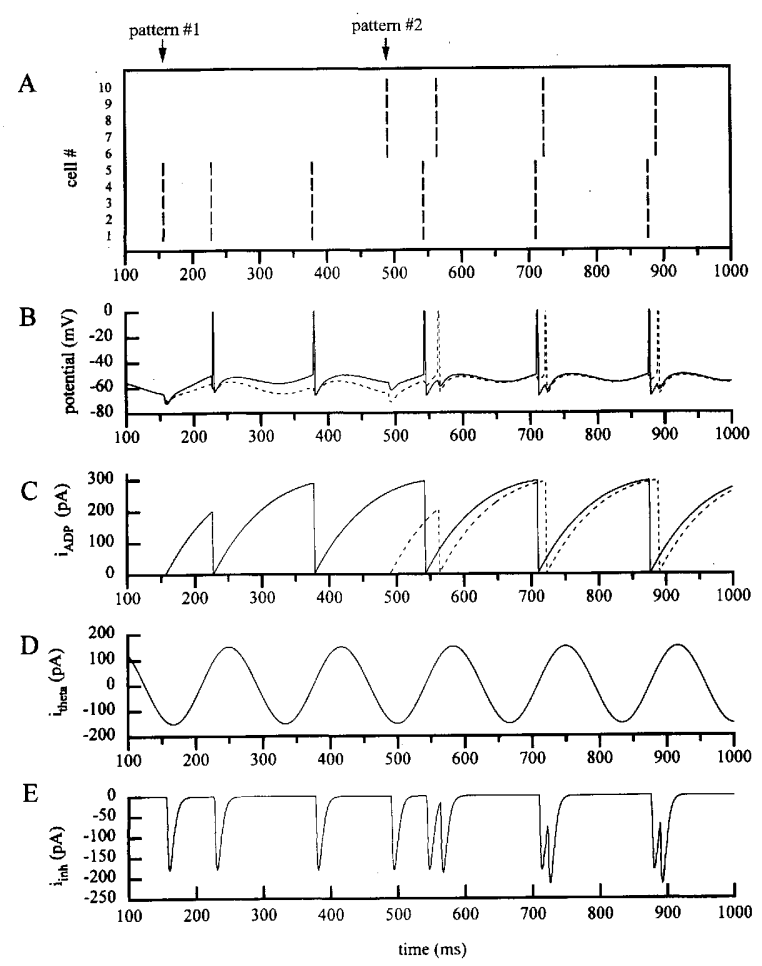

Figure 2: Mechanism of STM network (Fig. 1A). The first memory pattern is introduced at $t=160 \mathrm{msec}$. The solid line in $(B)$ is the membrane potential from cell 1 that encodes pattern 1 . The broken line is the membrane potential from cell 6 that encodes pattern 2 . The ramp of excitation, the ADP, is shown for cell 1 and cell 6 in $C$. The spiking of an activated cell is maintained by the ADP and the frequency of the spiking is locked to the subthreshold theta input $(D)$. At $t=500 \mathrm{msec}$ the second pattern is introduced. As seen in $E$, the feedback inhibition keeps the memories separated in time.

1991; Bragin et al. 1995), entorhinal cortex (Green and Arduini 1954; Mitchell and Ranek 1980), the cingulate cortex (Leung and Borst 1987) and neocortex (Biedenback 1966; Silva et al. 1991; Nakamura et al. 1992). The medial septum provides both cholinergic and GABAergic projections to the entorhinal-hippocampal-entorhinal loop (Stewart and Fox 1990). Furthermore, temporal correlations between active cells in the septum and the hippocampal system indicate that not only does the septum provide a con stant cholinergic modulation that facilitates oscillations, but it also induces a phasic drive (Alonso et al. 1987). Regions in medial forebrain having the same oscillatory properties as the septum are known to project to cortical areas (Alonso et al. 1996). We imagine that the same type of projections drives certain cortical regions at theta fre-

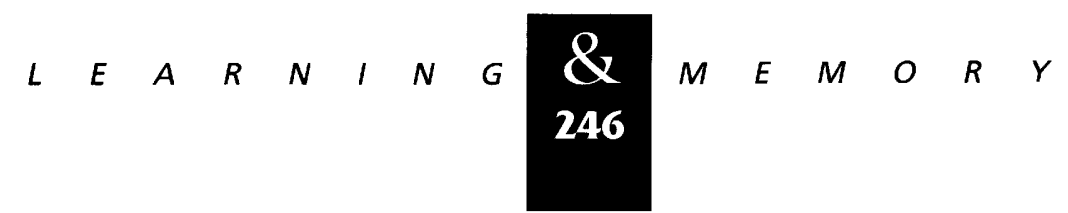


quency just as the septum drives the hippocampus. Hence the oscillatory input to cortex and hippocampus is modeled as

$$
i_{\text {theta }}(t)=B_{\text {tbeta }} \sin (2 \pi f t)
$$

where the theta frequency $f=6 \mathrm{~Hz}$.

\section{FEEDBACK INHIBITION}

In general, interneurons have been found to provide a powerful inhibitory feedback to the pyramidal cells, (Andersen et al. 1963; Andersen et al. 1964; Cobb et al. 1995). Furthermore, intracellular recordings, in vivo, from basket cells in the rat hippocampus show that these cells fire at gamma frequency (Sik et al. 1995) on the positive portion of the theta oscillation. Place cell recordings of rats show that interneurons fire at a much higher frequency than the pyramidal neurons, and that they have no spatial selectivity (Jung et al. 1994). This suggests that the inhibitory feedback could be responsible for generating the gamma oscillations in the following way: The firing of a subset of the pyramidal neurons will excite the entire interneuronal network through converging excitatory inputs. The interneurons will then provide an inhibitory feedback to all pyramidal cells. After the inhibition wears off, a new subset of pyramidal neurons will become active and so forth. Because we assume that all interneurons fire in synchrony we simply model the net GABAergic input as

$i_{G A B A}(t)=$

$$
\frac{A_{G A B A}}{a N} \sum_{j}^{N}\left(\frac{t-t_{\text {fire }}^{j}}{\tau_{G A B A}}\right) \exp \left(1-\frac{t-t_{\text {fire }}^{j}}{\tau_{G A B A}}\right)
$$

where $t^{j}$ refers to the action potential of the $j$ th pyramidal cell. We have chosen $\tau_{G A B A}=4 \mathrm{msec}$ (Miles 1990). The term $a N$ serves to normalize the GABAergic input if the network size is changed. $N$ is the number of pyramidal neurons in the network. The constant $a$ denotes the sparseness of pyramidal cells coding for information (i.e., $a=M / N$ where $M$ is the number of cells representing a pattern). In these papers we always $\operatorname{keep} M$ constant: $M=a N=5$.

\section{SYNAPTIC INPUT}

The recurrent collaterals are all excitatory and make one to one connections with all of the pyramidal cell. The synaptic transmission is mediated by synaptically released glutamate binding to AMPA and NMDA receptors. The synaptic input to cell $i$ is

$$
i_{s y n}^{i}(t)=i_{A M P A}^{i}(t)+i_{N M D A}^{i}(t)
$$

where the AMPA current is modeled by an alphafunction

$$
\begin{aligned}
i_{A M P A}^{i}(t)= & \frac{A_{A M P A}}{a N} \sum_{j}^{N} w_{\text {syn }}^{i j}\left(\frac{t-t_{\text {fire }}^{j}-t_{\text {delay }}}{\tau_{\text {AMPA }}}\right) \\
& \exp \left(1-\frac{t-t_{\text {fire }}^{j}-t_{\text {delay }}}{\tau_{\text {AMPA }}}\right)
\end{aligned}
$$

and the NMDA current by a double exponential

$$
\begin{aligned}
& i_{N M D A}^{i}(t)= \\
& \frac{A_{N M D A}}{a N} \sum_{j}^{N} w_{\text {syn }}^{i j} \exp \left(-\frac{t-t_{\text {fire }}^{j}-t_{\text {delay }}}{\tau_{N M D A, f}}\right) \\
& \left(1-\exp \left(-\frac{t-t_{\text {fire }}^{j}-t_{\text {delay }}}{\tau_{N M D A, r}}\right)\right)
\end{aligned}
$$

here $w_{s y n}^{i j}$ is the relative amplitude of an EPSC in cell $i$ evoked by cell $j$. For the time course of the AMPA-mediated EPSCs we have chosen $\tau_{A M P A}=1.5 \mathrm{msec}$ (Williams and Johnston 1991). In this paper (but not in Jensen and Lisman 1996a,c, this issue) we assume that only AMPA receptors participate in producing the EPSP; $A_{N M D A}=0 \mathrm{~mA}$. However the fall and rise time for the NMDA component is of importance for synaptic modification, $\tau_{N M D A, f}=7.0 \mathrm{msec}$ and $\tau_{N M D A, r}=1.0 \mathrm{msec}$. The delay in the recurrent feedback is $t_{\text {delay }}=0.5 \mathrm{msec}$.

\section{SYNAPTIC MODIFICATION}

The repetition of memory patterns in the short-term memory buffer allows us to consider

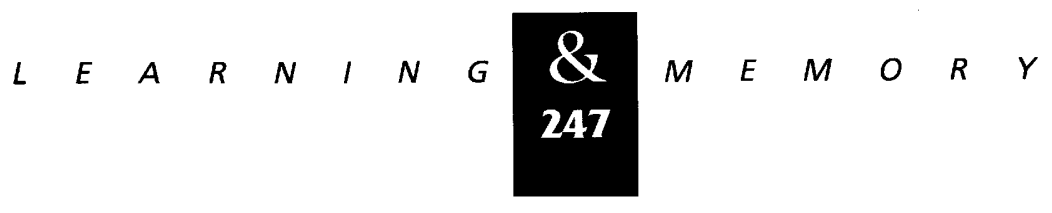


physiological realistic learning rules. Long-term potentiation (LTP) is a possible physiological implementation of the Hebb-rule: Simultaneous preand postsynaptic activity enhances synaptic efficacy. The enhancement has been shown to depend on NMDA receptors that are activated by the binding of glutamate. The activated NMDA receptors allow an inward current of $\mathrm{Ca}^{2+}$, provided there is sufficient postsynaptic depolarization. Through less understood mechanisms, the $\mathrm{Ca}^{2+}$ rise triggers LTP by increasing AMPA- and NMDA-mediated synaptic responses (for review, see Bliss and Collingridge 1993). We assume that the postsynaptic depolarization is attributable to back-propagating action potentials or other dendritic depolarizing events that occur with a small delay relative to the spike initiation in the somatic region (Markram et al. 1995):

$$
i_{\text {post }}(t)=\frac{t}{\tau_{\text {post }}} \exp \left(1-\frac{t}{\tau_{\text {post }}}\right)
$$

where $\tau_{\text {post }}=2.0 \mathrm{msec}$ is the duration of a backpropagating spike at the postsynaptic spine.

As mentioned, the kinetics of the NMDA channels is going to be crucial for the function of the network. We model the time course of the glutamate bound to the NMDA receptors by a double exponential:

$$
b_{g l u}(\mathrm{t})=\exp \left(-\frac{t}{\tau_{N M D A, f}}\right)\left(1-\exp \left(-\frac{t}{\tau_{N M D A, r}}\right)\right)
$$

We apply the values $\tau_{N M D A, f}=7.0 \mathrm{msec}$ and $\tau_{N M D A, r}=1.0 \mathrm{msec}$ to characterize the kinetics of the fast NMDA receptors (Monyer et al. 1994; S. Vicini, J.F. Wang, J.S. Poling, and D.R. Grayson, pers. comm.; see Discussion). Finally, the synaptic strength from cell $i$ to cell $j$ is determined by:

$$
\begin{aligned}
& \frac{d w_{s y n}^{i j}}{d t}= \\
& \frac{i_{\text {post }}\left(t-t_{\text {fire }}^{j}\right) \cdot b_{\text {glu }}\left(t-t_{\text {fire }}^{i}-t_{\text {delay }}\right)}{\tau_{p p}}\left(1-w_{s y n}^{i j}\right) \\
& \quad+\left(\frac{i_{\text {post }}\left(t-t_{\text {fire }}^{j}\right)}{\tau_{\text {npp }}}+\frac{b_{\text {glu }}\left(t-t_{\text {fire }}^{i}-t_{\text {delay }}\right)}{\tau_{p n p}}\right) \\
& \left(0-w_{s y n}^{i j}\right)
\end{aligned}
$$

where $\tau_{\text {delay }}$ is the time it takes an action potential to travel from the soma to the synapses of the recurrent collaterals. The first term in the expression is responsible for implementing Hebbian LTP. If $b_{g l u}(t)$ and $i_{\text {post }}(t)$ peak at the same time, $w_{i j}$ will approach one with the characteristic time $\tau_{p p}$ (assuming $\tau_{p p}<<\tau_{n p p}$ and $\left.\tau_{p p}<<\tau_{p n p}\right)$. If the preand postsynaptic events are slightly asynchronous, the potentiation is graded with respect to the temporal separation of the events. If the events are fully asynchronous a decrease in synaptic efficacy occurs: LTD (for review, see Christie et al. 1994; Linden 1994). This is modeled by the second term. If a presynaptic event occurs without a postsynaptic event, the synaptic weight $w_{i j}$ approaches zero with a characteristic time $\tau_{p n p}$ (pre-, not post-). Similar if only a postsynaptic event occurs, $w_{i j}$ approaches zero with the time $\tau_{n p p}$ (not pre-, post-). We apply the values $\tau_{p p}=50$ msec and $\tau_{p n p}=\tau_{n p p}=250 \mathrm{msec}$. Note that the learning rule we use causes the value at which synapses saturate to be graded with respect to the convolved pre- and postsynaptic activity no matter how many times stimulation is repeated.

NOISE

As a computational convenience we add Gaussian noise to the firing threshold of each pyramidal cell at each gamma cycle. The standard deviation is $\sigma=0.05 \mathrm{mV}$.

\section{EXTERNAL INPUT}

The external input $\boldsymbol{i}_{\text {ext }}$ serves to excite pyramidal neurons when memories are introduced. In the simulations we always introduce novel patterns at the troughs of theta cycles.

We have used the constants $A_{A D P}=300 \mathrm{pA}$, $B_{\text {theta }}=150 \mathrm{pA}, A_{A}=-120 \mathrm{pA}, A_{G A B A}=-180 \mathrm{pA}$ and $A_{A M P A}=700$ pA. Slightly different parameters are used in some of the simulations in Jensen and Lisman (1996a,b,c this issue). See figure captions for details. When calculating the membrane potentials numerically we use the time step $\Delta t=0.1$ msec. In the simulations we use a network of 10 to 40 cells. We use relatively few cells in our simulations for the sake of fast simulations.

\section{Results}

\section{GENERAL PROPERTIES OF NETWORK FUNCTION}

Before proceeding with issues related to LTM, it will be useful to describe how the STM network

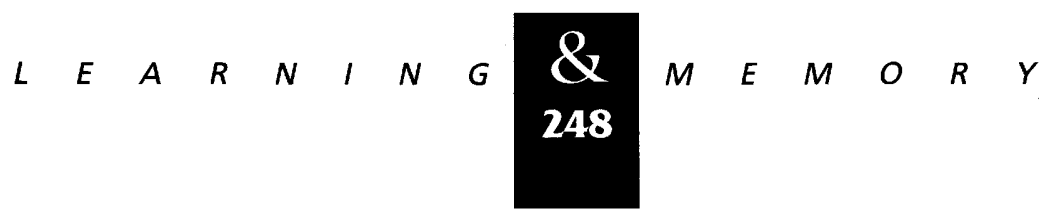


acts as a buffer to capture multiple items as they occur in real time. An STM is represented by the coincident firing of a subset of neurons within a particular gamma cycle. Different memories are encoded by different subsets. In Figure $2 \mathrm{~A}, \mathrm{~B}$ one memory is inserted into the buffer at $160 \mathrm{msec}$ by an external input that synchronously fires five of the cells shown. This firing triggers a slowly rising ADP. The ADP causes the cells to fire on the subsequent theta cycle (Fig. 2A,B). When this happens, the ADP ramp is reset (refreshed) making it possible for the same processes to occur on the next theta cycle. A second memory is inserted 340 msec after the first in the trough of the theta cycle. This leads to synchronous firing of two other cells. On subsequent theta cycles, the dynamics of the network lead to the firing of this memory in the second gamma cycle. Gamma cycles are separated by $12 \mathrm{msec}(80 \mathrm{~Hz})$ in this simulation. The fact that the memories occurred $340 \mathrm{msec}$ apart, but are recreated with a separation of $12 \mathrm{msec}$, is what is termed time compression (Tank and Hopfield 1987; Skaggs et al. 1996). Because multiple memories are actively maintained in different time slots, we refer to this as a multiplexed STM buffer.

The function of the network can be understood in terms of the component currents of the pyramidal cells (Fig. 2C-E). The ADP causes persistent firing and controls the timing of the firing. In the right part of Figure $2 \mathrm{C}$ it can be seen that the ramps for the two memories are slightly offset in time and it is this offset which brings cells that encode the first memory to threshold before cells that encode the second (within each theta cycle).
Figure 2E shows the feedback inhibition that follows each action potential. It is this inhibition that serves to restrict firing to discrete phases of the theta oscillation.

The order in which a set of memories is repeated in the STM buffer is independent of the exact phase of introduction of new memories. Figure 3 shows what happens when a fourth memory is presented to a network that already contains three active memories. As can be seen, the fourth memory is always stored in the fourth gamma cycle. Over a certain range, the exact phase of introduction is not important (phases during active firing of memories already in the buffer were not examined). It would seem to be important that a new memory is not introduced during the period that previously inserted memories are active and we imagine that a network earlier in the sensory pathway has the capability of storing information for up to several hundred milliseconds and controlling the entry of information into the shortterm buffer. The discretization of sensory processing by oscillations, even at the level of the retina (Neuenschwander and Singer 1996) and the very short-term memory processes termed iconic (Long 1980) and echoic memory present in primary sensory cortex (Wickelgren 1969; Lu et al. 1992) may fulfill these requirements.

LIFETIME OF NMDA CHANNELS COMPARED WITH THE DURATION OF A GAMMA CYCLE

We now consider how LTM formation would occur if the STM buffer described above had re-
A

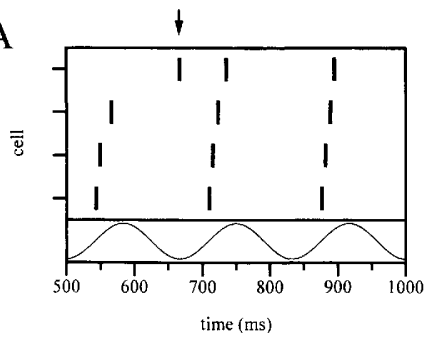

C

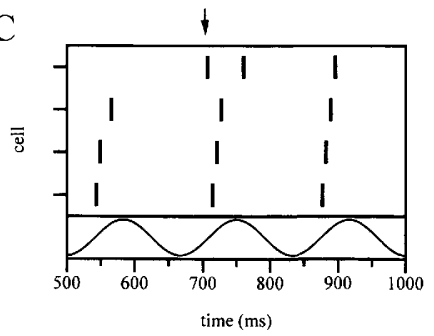

B

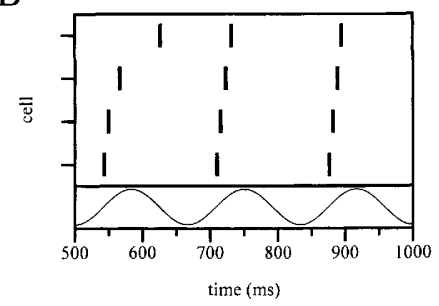

$\mathrm{D}$

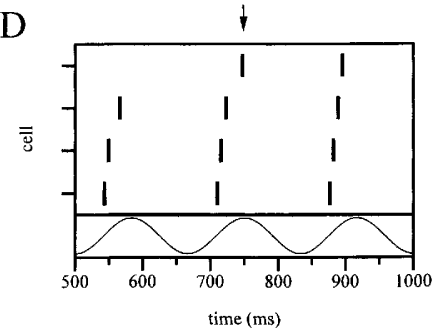

Figure 3: The order of memories in the STM buffer is independent of the exact phase at which they are introduced. A new memory (\#4) is introduced to the network at different phases. As seen, the new memory always ends up being the last to be activated in the buffer (i.e., the order of the memory items in the STM is independent of when they are presented). The new memory is introduced at the phases $0,270,90$, and 170 . Introduction of new memory during the activity of an old memory was not considered (see text).

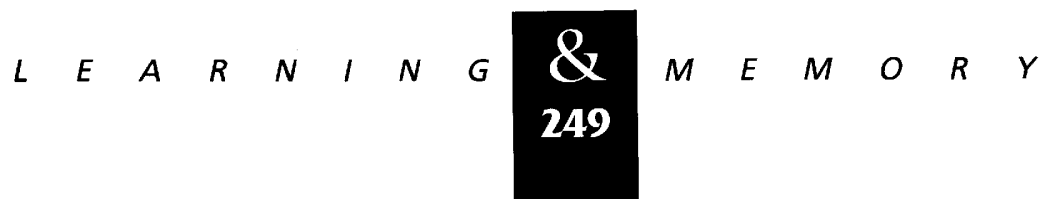


current connections with modifiable synapses. We assume that all principal neurons are interconnected by recurrents (Fig. 1C). Physiological work on Hebbian synapses allows us to predict what will happen at these recurrent synapses. Although as stated by Hebb, synchronous firing in the presynaptic and postsynaptic cell is the condition for strengthening synapses, the exact meaning of synchrony was not defined. Experiments show that LTP can occur even when the postsynaptic depolarization occurs with a delay after presynaptic firing (Levy and Steward 1983; Gustafsson and Wigström 1986; Gustafsson et al. 1987; Larson and Lynch 1989). The time constant of deactivation of the NMDA channels determines how long this delay can be.

The dependence of synaptic modification ( $\Delta w$ ) on the timing of pre- and postsynaptic activity in theta/gamma networks is illustrated in Figure 4 . When memory 1 is activated, glutamate is released from the axons of the pyramidal cells coding for memory 1 . The glutamate binds to the NMDA receptors on all postsynaptic pyramidal cells and leads to an activated state (Fig. 4B1). Channels in the activated state can open, but only if the postsynaptic cell depolarizes to relieve the $\mathrm{Mg}^{2+}$ block. When opening occurs, there is an influx of $\mathrm{Ca}^{2+}$ (not modeled explicitly) which leads to LTP. This increases the synaptic strength by a value $\left(\Delta w_{i j}\right)$ proportional to the convolution of the time course of the activated state of the NMDA channels and the postsynaptic depolarization (see Material and Methods).

Figure 4 shows that the synaptic connections that are strengthened in a theta/gamma network depend critically on the time constant of deactivation of NMDA channels (unbinding of glutamate). If the NMDA channels have fast deactivation and therefore span only one gamma cycle (Fig. 4B1), then cells that fire in different gamma cycles (Fig. 4A) and therefore represent different memories, will not have the synapses between them strengthened (Fig. $4 \mathrm{~B} 2, \Delta w_{i j}$ ). The only connections that are strengthened are between cells that represent the same memory. The synapses that are strengthened are thus appropriate for autoassociative LTM. If however, NMDA channels have a slow deactivation that spans several gamma cycles (Fig. 4C1), connections would be strengthened between cells that represent different memories (Fig. 4C2). This is what we call heteroassociative memory. In this paper we will assume that

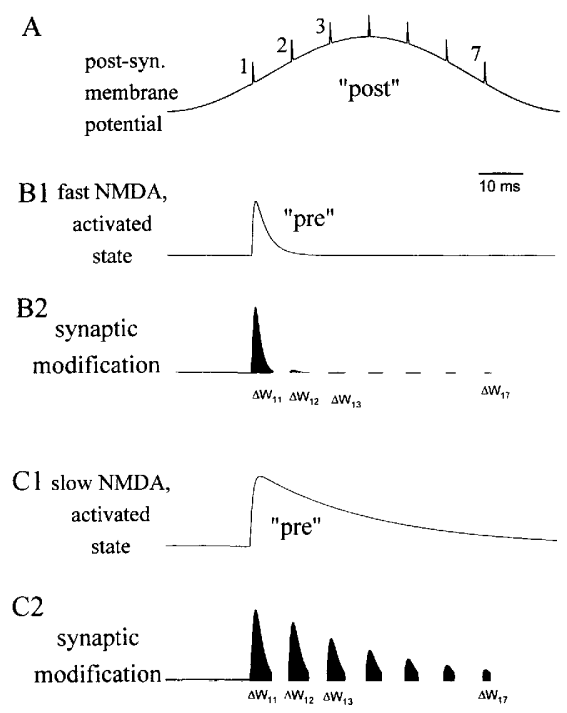

Figure 4: Rules for LTP induction indicate that the type of memory formed in networks with theta/gamma oscillations will depend on the time constant of the NMDA channel. (A) Representation of the seven different memories becoming active within a theta cycle. (B1) The time course of fast NMDA channels activated by memory item one. (B2) Strong connections form only between cells that fire in the same gamma cycle. Because these cells encoded the same memory, the fast NMDA channel forms the connections needed for autoassociation. $\Delta w_{i j}$ represents the synaptic connections between cell $i$ and $j$. (C1) The time course of slow NMDA channels whose time constant spans multiple gamma cycles. (C2) Connections form between memory item one and subsequent memories (i.e., heteroassociations are formed)

the network contains only fast NMDA channels and is therefore specialized for the formation of autoassociative LTM.

\section{FORMATION OF LTM FOR NOVEL ITEMS}

We now consider how a novel autoassociative memory can be incorporated into LTM in a physiologically realistic way in a network with fast NMDA channels. This physiological realism includes the assumption that synaptic modification requires substantial repetition (see Introduction, Materials and Methods). We also have added random membrane noise not present in our previous simulations (Figs. 2 and 3; Lisman and Idiart 1995).

Our simulation in Figure 5 shows the formation of LTM for multiple items presented in real

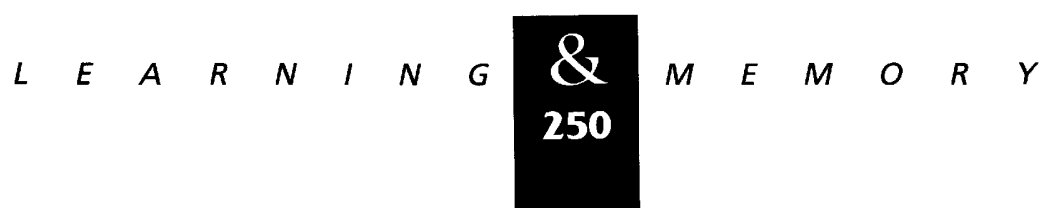


A

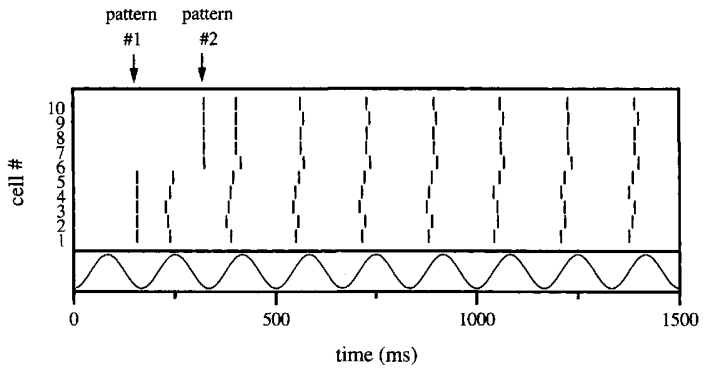

B

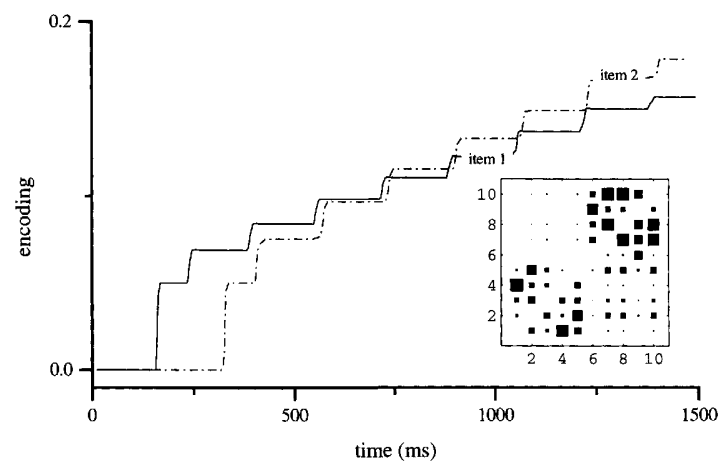

Figure 5: (A) Two novel memory patterns are introduced to the network on subsequent theta cycles. Cells 1-5 code for pattern 1 and cells $6-10$ code for pattern 2 . At each subsequent theta cycle the two memories become reactivated, but are degraded because of noise (become unsynchronized within the gamma cycles). (B) With a physiological learning rule, LTM for the two memories builds up slowly. The synaptic encoding is a measure of the actual synaptic values relative to synaptic values corresponding to perfect encoding. Because of noise the memories are degraded and the errors are encoded, as illustrated by the inset representing the synaptic matrix. The cell number (1-10) is specified on both axes; the size of the square at location $i, j$ denotes the synaptic strength of the connection between cell $i$ ( $y$ axis) and cell $j$ (x-axis).

time to the network. In the example, two novel memories are presented to the network (arrows in Fig. 5A). The ADP leads to the firing of both memories on subsequent theta cycles, but within a few cycles, the firing is progressively desynchronized. This is because the membrane noise generates errors in the ADP timing ramps and causes cells to fire in the wrong gamma subcycle. Because the ADP that controls timing is unitary, there is no basis for error correction. Thus, once a timing error occurs for a cell, the error continues on subsequent theta cycles. As seen in Figure 5B, the repetition of memories in STM gradually leads to the build up of synaptic strength in LTM. The memory information is thus learned. However, to the extent that some cells participating in a memory representation eventually fire in the wrong gamma cycle, errors will also become synaptically encoded. This is illustrated by the inset in Figure $5 B$. The size of each matrix element represents the strength of the synapse from cell $i$ to cell $j$. We conclude that novel memories can be incorporated into LTM after a single presentation, but the encoding will inevitably contain inaccuracies.

Although a novel item cannot accurately be encoded into LTM by a single presentation, we thought that multiple presentations of the same sequence might lead to correct encoding if erroneous information stored during a previous presentation could be removed during a subsequent presentation by LTD (for review, see Christie et al. 1994; Linden 1994; Debanne and Thompson 1996). We model LTD by decreasing the synaptic conductance if a presynaptic activation occurs without postsynaptic activation or vice versa. The time window for which the pre- and postsynaptic events are considered synchronous is determined by the time characteristic of the NMDA channel (see Materials and Methods). Hence, if some synapses had been erroneously strengthened, one could expect that reintroduction of the memory patterns will reduce the erroneously strengthened synapses. This is, however, not the case if the same sequence of memory patterns is repeatedly presented. Some of the synapses that were erroneously strengthened during initial presentations do not get weakened (Fig. 6A).

Correct encoding can occur if the order of the same memory patterns is varied from presentation to presentation. We have applied this procedure in Figure 6B. Initially, some errors were encoded into the synaptic matrix. After the sequence was presented repeatedly with the order interchanged, a correct memory trace builds up.

\section{Discussion}

\section{MECHANISM OF STM}

It has been proposed that STM could rely on reverbatory mechanisms (Hebb 1949), and that the recurrent connections of attractor network are an example of such a reverbatory loop (Amit 1989). Specifically, in attractor networks activity is maintained because cells that represent a memory selectively excite each other with strong syn-

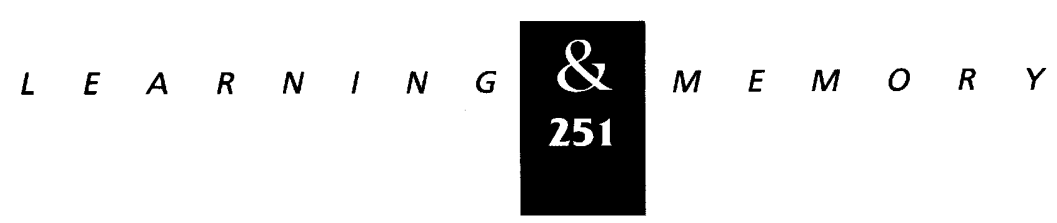


Jensen et al.

A

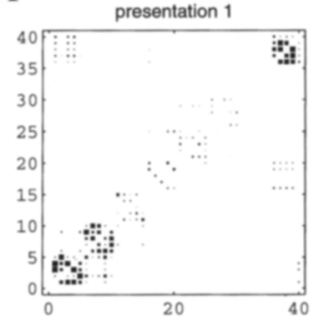

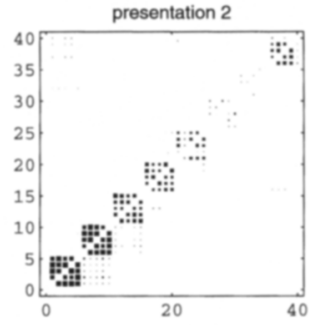

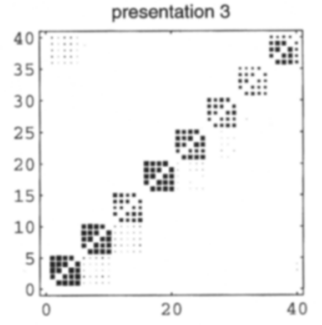

Figure 6: Conditions required for establishing error-free autoassociative LTM of individual items. Memories are presented in rapid succession during a learning period. Such presentations are repeated many times and the resulting synaptic weight matrix is shown as a function of the number of presentations. The matrix elements of $1-5$ on the $x$ - and $y$-axis represents the synapses ideally coding for the first memory. The next memory is ideally coded by the elements at 6-10 at the $x$ and $y$-axis and so forth. $(A)$ If the same eight memories are always introduced in the same order, the memories are never accurately learned, as shown by the iriappropriate components of the matrix. (B) If the eight memories are introduced in random order, the matrix becomes accurate. The simulations were done the following way: A sequence of items was selected from a eight-item list and introduced to the system. The sequence was kept active for 20 theta cycles allowing the individual memories to become encoded into the synapses. Then a new sequence was selected and introduced to the network. In $A$ seven consecutive items was select from a list of eight elements and introduced to the network. In $B$ the seven items were selected randomly from the list before each introduction. The final synaptic values ranged from 0 to 0.8 .
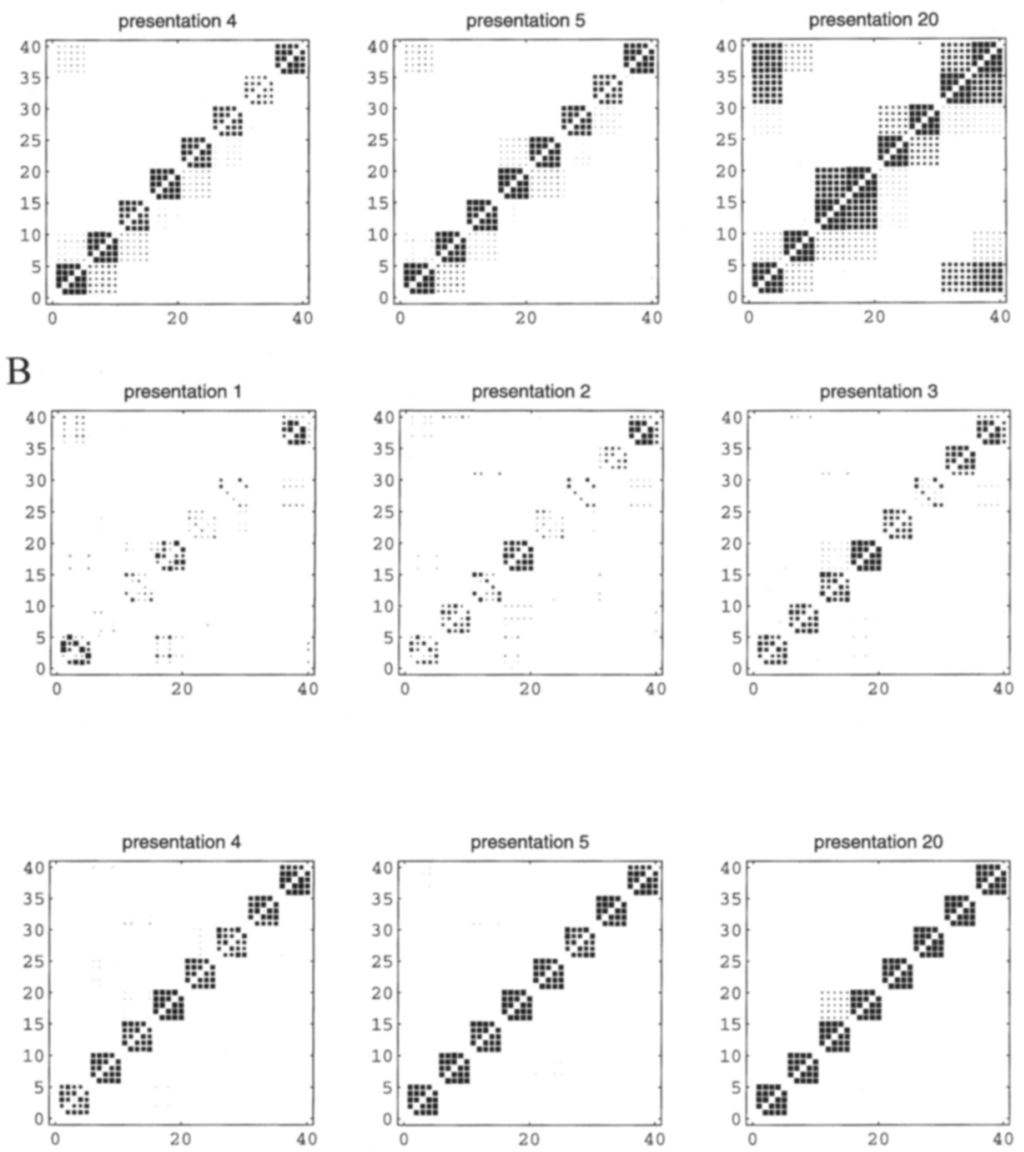

apses. By definition, this selectivity cannot exist for novel memories. Thus, if repeated firing in STM is necessary to produce LTM of novel memories, the firing cannot be produced by recurrent connections. This leaves intrinsic membrane changes (such as the ADP) as the only known possibility for sustaining firing. This mechanism is nonredundant and thus cannot be error correcting. We conclude that STM for novel items is necessarily inaccurate. The degree of inaccuracy will depend on the detailed membrane properties and the level of cellular noise. We are unable to estimate how fast
STM will be disrupted by these factors. The fidelity of STM for known items is much greater, as will be described in the next paper (Jensen and Lisman $1996 \mathrm{~b}$, this issue).

\section{REPETITION BY STM INCORPORATES NOVEL ITEMS INTO LTM}

Our results show that an integrated STM and LTM network can capture multiple, novel memory items in real time and incorporate them 
into LTM. The STM mechanism produces a repetition of each item once every theta cycle and thereby leads to the gradual incorporation of each item into LTM (Fig. 5). This repetition is crucial because physiological analysis has shown that the synaptic modifications that underlie LTM occur slowly (Larson et al. 1986; Hanse and Gustafsson 1994).

To the extent that errors occur in STM because of noise, as discussed above, the LTM that is slowly formed will be inaccurate (Fig. 5). There appears to be an interesting optimization problem here: The longer STM is maintained, the greater the encoding into LTM. However, the longer STM is maintained, the greater the chance for the corruption of information by noise. We cannot determine where the optima lies, but what is clear is that the encoding of autoassociative LTM will necessarily be inaccurate after a single presentation.

Figure 6 shows that accurate encoding can be achieved if multiple presentations are given. Each time the item is presented again, different errors will occur and some of the previous errors will be erased by activity-dependent depression of synapses (Fig. 6B). Eventually, a reasonably accurate LTM is formed by a kind of averaging process. One more generalization should be noted. If a novel memory item is always presented in the same temporal context (i.e., with the same items preceding and succeeding), erroneously encoded patterns tend to build up rather than get averaged out (Fig. 6A). To avoid this a novel memory item must appear in different temporal context with each presentation. This makes intuitive sense. If we are learning new words of a foreign language and a set of words always appears together in the same order, we will not be able to learn each word as an individual item. Only if the novel words appear separately, interleaved in different contexts, will we be able to extract them as single entities. McClelland et al. (1995) also argue for the importance of interleaved learning.

\section{ROLE FOR FAST NMDA CHANNELS}

The study of NMDA channels has shown that different NMDA channels have different kinetics (Hestrin et al. 1992; Monyer et al. 1994; S. Vicini, J.F. Wang, J.S. Poling, and D.R. Grayson, pers. comm.) Recombinant NMDA receptors composed by the NMDAR1a subunit combined with the NMDAR2A subunit have a much faster deactiva- tion time constant ( $\cong 36 \mathrm{msec}$ ) than receptors with $2 \mathrm{~B}$ or $2 \mathrm{C}$ subunits ( $\cong 300 \mathrm{msec})$. These time constants are measured at room temperature and will be much shorter at body temperature given a $Q_{10}$ of $\sim 3$ (Hestrin et al. 1990; Feldmeyer et al. 1993). NMDA channels with fast kinetics have been observed in cortical networks (Carmignoto and Vicini 1992; Monyer et al. 1994). The role of this diversity has been completely unclear from a computational point of view. This paper provides the first indication of the reason for this diversity and the particular function that fast NMDA channels might perform. Fast NMDA channels have time constants that do not span multiple gamma cycles. They will thus lead to autoassociative memory formation in networks with theta/gamma oscillations. The reader is referred to the Discussion section of the next paper in this series (Jensen and Lisman 1996b, this issue) for a discussion of other biological aspects of this model.

\section{Acknowledgments}

We thank Larry Abbott and Jean-Marc Fellous for reading the manuscript. This work was supported by the W.M. Keck Foundation, a National Institutes of Health grant (NS 27337) and the Alfred P. Sloan Foundation (94-10-1).

The publication costs of this article were defrayed in part by payment of page charges. This article must therefore be hereby marked "advertisement" in accordance with 18 USC section 1734 solely to indicate this fact.

\section{References}

Abeles, M., H. Bergman, E. Margalit, and E. Vaadia. 1993. Spatiotemporal firing patterns in the frontal cortex of behaving monkeys. I. Neurosci. 70: 1629-1638.

Alonso, A., J.M. Gaztelu, W. Buno Jr., and E. Garcia-Austt. 1987. Crosscorrelation analysis of septohippocampal neurons during theta-rhythm. Brain Res. 413: 135-146.

Alonso, A., A. Khateb, P. Fort, B.E. Jones, and Michel Mühlethaler. 1996. Differential oscillatory properties of cholinergic and non-cholinergic nucleus basalis neurons in guinea pig brain slice. Eur. J. Neurosci. 8: 169-182.

Amit, D.J. 1989. Modeling brain function: The world of attractor neural networks. Cambridge University Press, Cambridge, UK.

Andersen, P., J.C. Eccles, and Y. Løying. 1963. Recurrent inhibition in the hippocampus with identification of the inhibitory cell and its synapses. Nature 198: $540-542$.

- 1964. Pathway of postsynaptic inhibition in the hippocampus. J. Neurophysiol. 27: 608-619.

Andrade, R. 1991. The effect of carbachol which affect muscarinic receptors was investigated in prefrontal layer $V$ neurons. Brain Res. 541: 81-93.

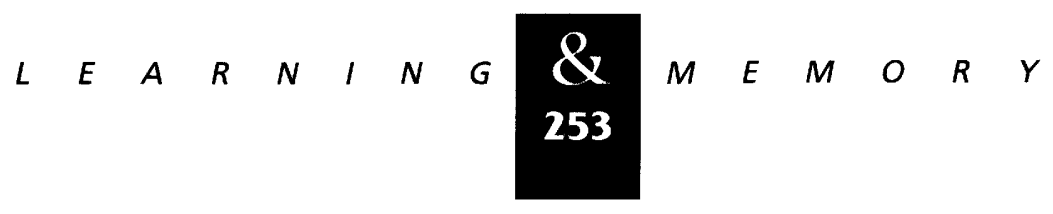


Jensen et al.

Araneda, R. and R. Andrade. 1991. 5-Hydroxytryptamine 2 and 5-hydroxytryptamine IA receptors mediate opposing responses on membrane excitability in rat association cortex. Neuroscience 40: 399-412.

Biedenbach, M.A. 1966. Effects of anesthetics and cholinergic drugs on prepyriform electrical activity in cats. Exp. Neurol. 16: 464-479.

Bliss, T.V.P. and T. Lømo. 1973. Long-lasting potentiation of synaptic transmission in the dentate area of the anaesthetized rabbit following stimulation of the perforant path. J. Physiol. 232: $331-356$.

Bliss, T.V.P. and G.L. Collingridge. 1993. A synaptic model of memory: Longterm potentiation in the hippocampus. Nature 361: 31-39.

Bragin, A., G. Jando, Z. Nadasdy, J. Hetke, K. Wise, and G. Buzsaki. 1995. Gamma $(40-100 \mathrm{~Hz})$ oscillations in the hippocampus of the behaving rat. I. Neurosci. 15: 47-60.

Bressler, S.L. 1984. Spatial organizatıon of EEGs from olfactory bulb and cortex. Electroenceph. Clin. Neurophysiol. 57: 270-276.

Caeser, M., D.A. Brown, B.H. Gahwiler, and T. Knopfel. 1993. Characterization of a calcium-dependent current generating a slow after depolarization of CA3 pyramidal cells in rat hippocampal slice cultures. Eur. I. Neurosci. 5: $560-569$.

Carmignoto, G. and S. Vicini. 1992. Activity-dependent decrease in NMDA receptor responses during development of the visual cortex. Science 258: 1007-1011.

Christie, B.R., D.S. Kerr, and W.C. Abraham. 1994. Flip side of synaptic plasticity: Long-term depression mechanisms in the hippocampus. Hippocampus 4: 127-135.

Cobb, S.R., E.H. Buhl, K. Halasy, O. Paulsen, and P. Somogyi. 1995. Synchronization of neuronal activity in hippocampus by individual GABAergic interneurons. Nature 378: 75-78.

Debanne, D. and S.M. Thompson. 1996. Associative long-term depression in the hippocampus in vitro. Hippocampus 6: 9-16.

Feldmeyer, R., R.A. Silver, and G.C. Stuart. 1993. Temperature dependence of EPSC time course of the rat mossy fibre-granule synapses in thin cerebellar slices. J. Physiol. 459: 481.

Forsythe, I.D. and G.L. Westbrook. 1988. Slow excitatory postsynaptic currents mediated by $\mathrm{N}$-methyl-D-asparate receptors on cultured mouse central neurons. J. Physiol. 396: 515-533.

Fuster, J.M. and J.P. Jervey. 1982. Neuronal firing in the inferotemporal cortex of the monkey in a visual memorytask. J. Neurosci. 2: 361-375.
Gertsner, W., R. Ritz, and J.L. van Hemmen. 1993. Why spikes? Hebbian learning and retrieval of time-resolved excitation patterns. Biol. Cybernet. 69: 503-515.

Goldmann-Rakic, P.S. 1995. Cellular basis of working memory. Neuron 14: 477-486.

Gray, C.M., P. Konig, A.K. Engel, and W. Singer. 1989. Oscillatory responses in cat visual cortex exhibit intercolumnar synchronization which reflects global stimulus properties. Nature 338: 334-337.

Green, J.D. and A. Arduini. 1954. Hippocampal electrical activity in arousal. J. NeuroPhysiol. 17: 533-557.

Gustafsson, B. and H. Wigström. 1986. Hippocampal long-lasting potentiation produced by pairing single volleys and brief conditioning tetani evoked in separate afferents. I. Neurosci. 6: 1575-1582.

Gustafsson, B., H. Wigström, W.C. Abraham, and Y.Y. Huang. 1987. Long-term potentiation in the hippocampus using depolarizing current pulses as the conditioning stimulus to single volley synaptic potentials. J. Neurosci. 7: 774-780.

Hanse, E. and B. Gustafsson. 1994. Onset and stabilization of NMDA receptor-dependent hippocampal long-term potentiation. Neurosci. Res. 20: 15-25.

Hebb, D.O. 1949. The organization of behavior. Wiley, New York, NY.

Hertz, J., A. Krogh, and R.G. Palmer. 1991. Introduction to the theory of neural computation. Addison-Wesley, Redwood City, CA.

Hestrin, S. 1992. Developmental regulation of NMDA receptor-mediated synaptic currents at a central synapse. Nature 357: 686-689.

Hestrin, S., P. Sah, and R.A. Nicoll. 1990. Mechanisms generating the time course of dual component excitatory synaptic currents recorded in hippocampal slices. Neuron 5: $247-253$.

Hopfield, J.J. 1982. Neural networks and physical systems with emergent collective computational abilities. Proc. Nat/. Acad. Sci. 79: 2554-2558.

Hopfield, J.J. and A.V. Hertz. 1995. Rapid local synchronization of action potentials: Toward computation with coupled integrate-and-fire neurons. Proc. Natl. Acad. Sci. 92: 6655-6662.

Jahr, C.E. and C.F. Stevens. 1990. A quantitative description of NMDA receptor-channel kinetic behavior. I. Neurosci. 10: $1830-1837$.

Jensen, O. and J.E. Lisman. 1996a. Hippocampal CA3 region 
predicts memory sequences: Accounting for the phase precession of place cells. Learn. \& Mem. (this issue).

1996b. Novel lists of $7 \pm 2$ known items can be reliably stored in an oscillatory short-term memory network: Interaction with long-term memory. Learn. \& Mem. (this issue).

1996c. Theta/gamma networks with slow NMDA channels learn sequences and encode episodic memory: Role of NMDA channels in recall. Learn. \& Mem. (this issue).

Jung, M.W., S.I. Wiener, and B.L. McNaughton. 1994. Comparison of spatial firing characteristics of units in dorsal and ventral hippocampus of the rat. J. Neurosci.

14: $7347-7373$

Kahle, J.S. and C.W. Cotman. 1989. Carbachol depresses the synaptic responses in the medial but not lateral perforant path. Brain Res. 482: 159-163.

Larson, L. and G. Lynch. 1989. Theta pattern stimulation and the induction of LTP: Sequence in which synapses are stimulated determines the degree to which they potentiate. Brain Res. 489: 49-58.

Larson, J., D.L. Wong, and C. Lynch. 1986. Patterned stimulation at the theta frequency is optimal for the induction of hippocampal long-term potentiation. Brain Res. 368: $347-350$.

Leung, L.-W.S. and J.G.G Borst. 1987. Electrical activity of the cingulate cortex. I. Generating mechanisms and relations to behavior. Brain Res. 407: 68-80.

Levy, W.B. and D. Steward. 1983. Temporal contiguity requirements for long-term associative potentiation/depression in the hippocampus. Neuroscience 8: 791-797.

Libri, V., A. Constanti, M. Calaminici, and G. Nistico. 1994. A comparison of the muscarinic response and morphological properties of identified cells in the guinea-pig olfactory cortex in vitro. Neuroscience 59: 331-347.

Linden, D.J. 1994. Long-term synaptic depression in the mammalian brain. Neuron 12: 457-472.

Lisman, J.E. and M.A.P. Idiart. 1995. Storage of $7 \pm 2$ short-term memories in oscillatory subcycles. Science 267: 1512-1515.

Long, G.M. 1980. Iconic memory: A review and critique of the study of short-term visual storage. Psychol. Bull. 88: $785-820$.

Lu, Z.L., S.J. Williamson, and L. Kaufman. 1992. Behavioral lifetime of human auditory sensory memory predicted by physiological measures. Science 258: 1668-1670.

McClelland, J.L., B.L. McNaughton, and R. O'Reilly. 1995. Why there are complementary learning systems in the hippocampus and neocortex: Insights from the successes and failures of connectionist models of learning and memory. Psychol. Rev. 102: 419-457

Markram, H., P.J. Helm, and B. Sakmann. 1995. Dendritic calcium transients evoked by single back-propagating action potentials in rat neocortical pyramidal neurons. J. Physiol. 485: $1-20$.

Miles, R. 1990. Synaptic excitation of inhibitory cells by single CA3 hippocampal pyramidal cells of the guinea-pig in vitro. J. Physiol. 428: 61-77.

Miller, G.A. 1956. The magical number seven, plus minus two: Some limits on our capacity for processing information. Psychol. Rev. 63: 81-97.

Mitchell, S.J. and J.B. Ranck. 1980. Generation of theta rhythm in medial entorhinal cortex of freely moving rat. Brain Res. 189: 49-66.

Monyer, H., N. Burnashev, D.J. Laurie, B. Sakmann, and P.H. Seeburg. 1994. Developmental and regional expression in the rat brain and functional properties of four NMDA receptors. Neuron 12: 529-540.

Morris, R.G., E. Anderson, G.S. Lynch, and M. Baudry. 1986. Selective impairment of learning and blockade of long-term potentiation by an N-methyl-D-aspartate receptor antagonist AP5. Nature 319: 774-776.

Nakamura, K, A. Mikami, and K. Kubota. 1992. Oscillatory neural activity related to visual short-term memory in monkey temporal pole. NeuroReport 3: 117-120.

Neuenschwander, S. and W. Singer. 1996. Long-range synchronization of oscillatory light responses in the cat retina and lateral geniculate nucleus. Nature 379: 728-732.

O'Keefe, J. and M.L. Recce. 1993. Phase relationship between hippocampal place units and the EEG theta rhythm. Hippocampus 3: 317-330.

Sik, A., M. Penttonen, A. Ylinen, and G. Buzsaki. 1995. Hippocampal CA1 interneurons: An in vivo intracellular labeling study. J. Neurosci. 15: 6651-6665.

Silva, L.R., Y. Amital, and B.W. Conners. 1991. Intrinsic oscillations of neocortex generated by layer 5 pyramidal neurons. Science 251: 432-435.

Skaggs, W.E., B.L. McNaughton, M.A. Wilson, and C.A. Barnes. 1996. Theta phase precision in hippocampal neuronal populations and the compression of temporal sequences. Hippocampus 6: 149-172.

Soltesz, I. and M. Deschenes. 1993. Low- and high-frequency membrane potential oscillations during theta activity in CAI and CA3 pyramidal neurons of the rat hippocampus under ketamine-xylazine anesthesia. /. Neurophys. 70: 97-116.

Sternberg, S. 1966. High-speed scanning in human memory. Science 153: 652-654. 
Jensen et al.

Stewart, M. and S.E. Fox. 1990. Do septal neurons pace the hippocampal theta rhythm? Neuron 13: 163-168.

1991. Hippocampal theta activity in monkeys. Brain Res. 538: 59-63.

Storm, J.F. 1989. An after-hyperpolarization of medium duration in rat hippocampal pyramidal cells. J. Physiol. 409: 171-190.

1990. Potassium currents in hippocampal pyramidal cells. Prog. Brain Res. 83: 161-187.

Tank, D.W. and J.J. Hopfield. 1987. Neural computation by concentrating information in time. Proc. Natl. Acad. Sci. 84: 1896-1900.

Turner, D.A. and P.A. Schwartzkroin. 1983. Electrical characteristics of dendrites and dendritic spines in intracellularly-stained $\mathrm{CA} 3$ and dentate neurons. J. Neurosci. 3: $2381-2394$.

Vanderwolf, C.H. 1969. Hippocampal electrical activity and voluntary movement in the rat. Electroenceph. Clin.

Neurophysiol. 26: $407-418$.

Wickelgren, W.A. 1969. Associative strength theory of recognition memory for pitch. J. Math. Psychol. 6: 13-67.

Williams, S.H. and D. Johnston. 1991. Kinetic properties of two anatomically distinct excitatory synapses in hippocampal CA3 pyramidal neurons. I. Neurophysiol. 66: 1010-1020.

Received June 25, 1996; accepted in revised form September 25, 1996. 


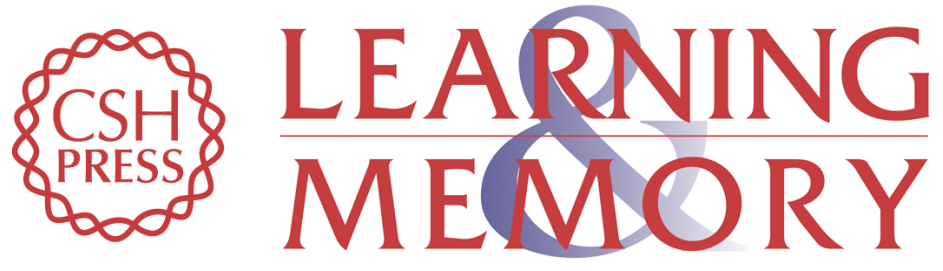

Physiologically realistic formation of autoassociative memory in networks with theta/gamma oscillations: role of fast NMDA channels.

O Jensen, M A Idiart and J E Lisman

Learn. Mem. 1996, 3:

References This article cites 67 articles, 16 of which can be accessed free at: http://learnmem.cshlp.org/content/3/2-3/243.full.html\#ref-list-1

License

Email Alerting Receive free email alerts when new articles cite this article - sign up in the box at the Service top right corner of the article or click here. 